\title{
Adsorptive Removal of Sulfamerazine from Water by modified Cellulose Nanofibers Aerogel
}

\author{
Qiufang Yao ${ }^{1}$, Bitao Fan ${ }^{1}$, Ye Xiong ${ }^{1}$, Chao Wang ${ }^{1}$, Qingfeng Sun ${ }^{1,2}$ \\ ${ }^{1}$ School of Engineering, Zhejiang A\&F University, Lin'an, China \\ ${ }^{2}$ Key Laboratory of Wood Science and Technology, Lin'an, China \\ E-mail: qfsun@zafu.edu.cn
}

\begin{abstract}
Keywords: Adsorption, sulfamerazine, graphene oxide, cellulose nanofibers aerogel Abstract. Using a chemical-ultrasonic process, a modified cellulose nanofibers aerogel, graphene oxide/cellulose nanofibers aerogel $(\mathrm{GO} / \mathrm{CNF})$ aerogel, was prepared, which was applied to adsorptive removal of sulfamerazine from water. The SEM, XRD and FTIR were used to characterize the $\mathrm{GO} / \mathrm{CNF}$. The as-prepared $\mathrm{GO} / \mathrm{CNF}$ had interconnected 3D network structure. The $\mathrm{GO} / \mathrm{CNF}$ could effectively removal sulfamerazine. Simultaneously, the GO/CNF still could be efficiently reused through five regeneration cycles.
\end{abstract}

\section{Introduction}

With rapid development of global economy and industry, there is increasingly serious pollution, especially water pollution. Aquatic ecosystems and human health are badly effect by organic micropollutants such as antibiotic and dye in water all over the world [1-5]. Therefore, effective governance over antibiotic pollution has become an important strategic objective of sustainable water resources utilization. To adapt to different antibiotic pollution cases, a large number of treatment technologies are widely used and conventional methods including such as adsorption, and degradation [6-8]. In these processes, with its simple, low cost, easy control and management and other features, the adsorption technology has been in the field of large-scale treatment application for a long time.

Cellulose is the most abundant natural biomass material on the earth, especially extracting from bamboo. The basic structure of this material has the advantage of forming many strong interactions with the filler particles or sheets and molding block of the aerogel from lots of powder-like materials, and this is the reason for thousands of bio products based on it. Here, cellulose nanofibers were used in our study.

Apart from graphene, GO exhibits different characteristic, such as good solvent dispersity, mainly due to the presence of oxygenated functional groups. Owing to these functional groups, GO has been widely used for pollutants removal studies and immobilizing numerous metallic nanoparticles [9]. However, until now GO had low surface area and poor removal performance compared to conventional activated carbons [10]. Recently, several studies have demonstrated that GO is excellently compatible within cellulosic matrix, It can be minimized by modifying the GO sheets with proper functional groups so as to maintain strong interactions with the cellulose matrix. In this work, we used grafting method to bond cellulose with GOs by using a one-pot ultrasonication method, providing a 3D network structure of $\mathrm{GO} / \mathrm{CNF}$.

\section{Material and methods}

Materials. Bamboo ( $>90 \%$ ) was recycled from Zhejiang A\&F University (Lin'an, China). Sulfamerazine (SM) was produced supplied by Aladdin Industrial Co. and its molecular structures were shown in Figure 1. 


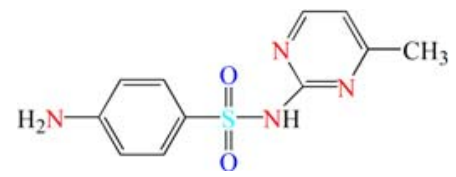

Figure 1 The molecular structures of SM

Preparation of graphene oxide (GO)

Graphene oxide was prepared from graphite powder (particle size lower than $30 \mathrm{um}$, Tianjin Kermel Chemical Regent Ltd, China) by a modified Hummers methods [11].

Fabrication of cellulose nanofibers (CNF) aerogel

The bamboo powder was purified to cellulose through some chemical methods. Then the purified cellulose was dispersed in distilled water, approximately $300 \mathrm{~mL}$ of the water slurry contained 0.1 $\mathrm{wt} \%$ samples. Thus, the purified cellulose microfibrils were split to thinner fibrils through ultrasonic treatment. The sonication then was conducted for 30 min with $500 \mathrm{~W}$ of an output power and freeze-dried under vacuum.

Synthesis of GO/CNF aerogel

The GO/CNF aerogel was prepared by the ultrasonication. In brief, $50 \mathrm{mg}$ CNF and GO was dissolved into $100 \mathrm{~mL}$ distilled water and stirred for $10 \mathrm{~min}$. Then the mixture suspension was placed in ice bath to be sonicated until the mixture liquid was homogeneous. Finally, the mixture liquid was freeze-dried under vacuum and used for adsorption.

Characterizations

The morphologies of the specimens were characterized by the scanning electron microscopy (SEM, FEI, Quanta 200, USA). Fourier transform infrared spectrum (FT-IR) measurements were carried out by using Nicolet 5700 Spectrometer in $\mathrm{KBr}$ pellet. Crystalline structures of the specimens were identified by the X-ray diffraction technique (XRD, Rigaku, D/MAX 2200, Japan) operating with $\mathrm{Cu} \mathrm{K \alpha}$ radiation $(\lambda=1.5418 \AA)$ at a scan rate $(2 \theta)$ of $2^{\circ} \mathrm{min}^{-1}$ and the accelerating voltage of 40 $\mathrm{kV}$. The concentrations of SM were determined by a UV-vis spectrophotometer (Model TU-1901). In detail, the concentrations of SM were determined by the UV-vis spectrophotometer performed at $266 \mathrm{~nm}$.

The adsorption of the antibiotics

The removal percentage ( $\mathrm{R} \%$ ) of antibiotics on the GO/CNF aerogel was calculated using Eq. (1). Simultaneously, Eq. (2) was used to calculate the mass of the adsorbed antibiotic per gram of the sorbent $\left(\mathrm{q}_{\mathrm{e}}, \mathrm{mg} / \mathrm{g}\right)$.

$$
\mathrm{R} \%=\left[\frac{\mathrm{C}_{0}-\mathrm{C}_{\mathrm{e}}}{\mathrm{C}_{0}}\right] \times 100 \%
$$

$$
\mathrm{q}_{\mathrm{e}}=\left[\frac{\mathrm{C}_{0}-\mathrm{C}_{\mathrm{e}}}{\mathrm{m}}\right] \times \mathrm{V}
$$

where $C_{o}$ is the initial antibiotic concentration $\left(\mathrm{mg} \cdot \mathrm{L}^{-1}\right), \mathrm{C}_{\mathrm{e}}$ is the equilibrium antibiotic concentration in solution $\left(\mathrm{mg} \cdot \mathrm{L}^{-1}\right), \mathrm{V}$ is the volume of solution $(\mathrm{L})$, and $\mathrm{m}$ is the weight of the $\mathrm{GO} / \mathrm{CNF}$ aerogel $(\mathrm{g})$.

The $0.04 \mathrm{~g} \mathrm{GO} / \mathrm{CNF}$ aerogel (content of GO within $60 \%$ ) was added to $20 \mathrm{~mL}$ of $20 \mathrm{mg} \cdot \mathrm{L}^{-1} \mathrm{SM}$ solution in Erlenmeyer flasks. The $\mathrm{pH}$ of the samples was adjusted to $\mathrm{pH} 2$. The $\mathrm{R} \%$ values of the six antibiotics were obtained at the conditions of $2 \mathrm{~g} \cdot \mathrm{L}^{-1}$ sorbent. In the reconstruction phase of the sorbent, the $\mathrm{GO} / \mathrm{CNF}$ is placed in contact with $20 \mathrm{~mL}$ of $\mathrm{NaOH} 8 \mathrm{w} / \mathrm{w} \%$ solution.

\section{Results and discussion}

Figure 2 showed the SEM images of the CNF and GO/CNF aerogel, respectively. The CNF aerogel, shown in Figure 2a, was present as individual flexible fibrils and partial cross-linked fibrils which its color was white and its density was $0.73 \mathrm{mg} / \mathrm{cm}^{3}$. For GO/CNF with $60 \%$ content of GO within the aerogels (Figure 2b), fewer single CNF fibrils were visible and almost only micro-sized sheets instead of individual and partial cross-linked CNF fibrils are visible which is ascribed to the enhanced binding between CNF by GO, which its color transformed grey and its density was $2.09 \mathrm{mg} / \mathrm{cm}^{3}$. 


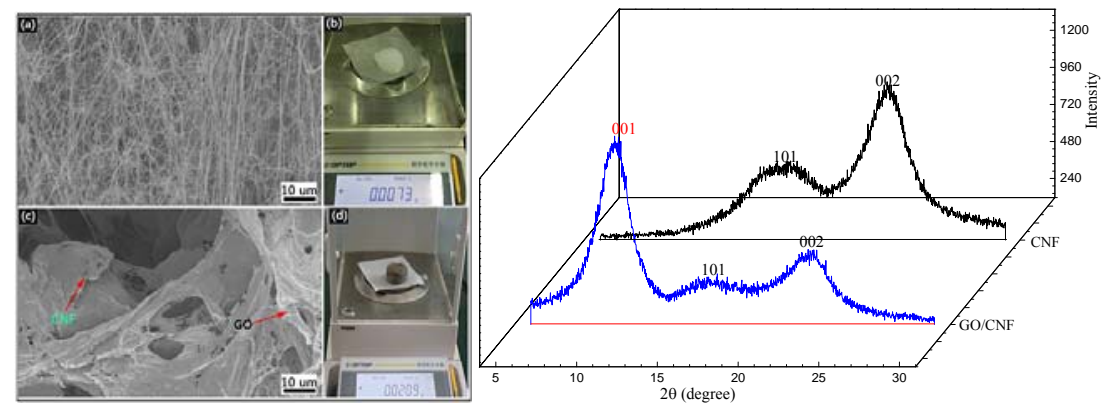

Figure 2 the SEM images and the interpolating photographs of the $\mathrm{CNF}$ and $\mathrm{GO} / \mathrm{CNF}$ aerogels, respectively (left)

Figure 3 the XRD patterns (a) of the $\mathrm{CNF}$ and the $\mathrm{GO} / \mathrm{CNF}$ (right).

Figure 3 presented the XRD patterns (a) and FTIR spectra (b) of the CNF and the GO/CNF. For the XRD patterns 3 (Figure $3 \mathrm{a}$ ), the diffraction peaks at about $22^{\circ}$ and $16^{\circ}$ were attributed to the typical reflection planes $(002)$ and (101) of bamboo cellulose I $[12,13]$. In XRD pattern of GO/CNF, an intense peak at $2 \theta=10.28^{\circ}$ can be observed, corresponding to the (001) diffraction planes, which is identical with reported values [13].

UV-vis spectrophotometer of different concentrations of SM and their standard curve were shown in Figure 4. A stock solution of the SM $\left(100 \mathrm{mg} \cdot \mathrm{L}^{-1}\right)$ was prepared by dissolving $0.1 \mathrm{~g}$ of SM in a liter of double distilled water. The other SM concentrations were prepared by diluting the stock solution with suitable volumes of double distilled water to form the concentrations of $1,2,5,8,10$, and 20 $\mathrm{mg} / \mathrm{L}$. By liner fit, the standard curve between the concentration and absorbance is shown in Figure 4
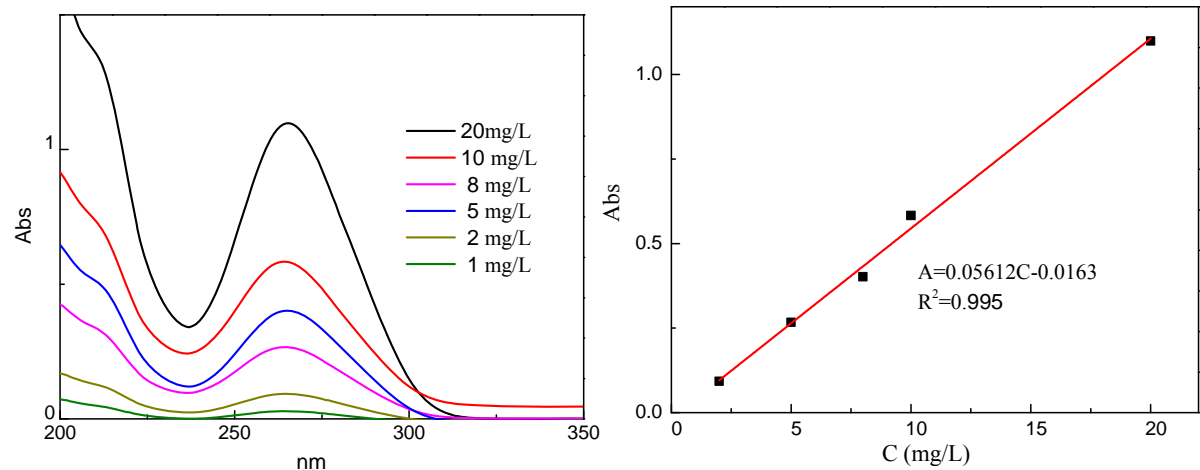

Figure $4 \mathrm{UV}$-vis spectrophotometer of different concentrations of SM and their standard curve (Left)

Figure 5 showed the adsorption kinetics, the correctional pseudo-first-order equation (3), and pseudo-second-order equation (4) kinetics models for adsorption of SM on GO/CNF (Right).

$$
\operatorname{In}\left(q_{e}-q_{t}\right)=\operatorname{Inq}_{e}-k_{1} t
$$

$$
\frac{\mathrm{t}}{\mathrm{q}_{\mathrm{t}}}=\frac{1}{\mathrm{k}_{2} \mathrm{q}_{\mathrm{e}}^{2}}+\frac{1}{\mathrm{q}_{\mathrm{e}}} \mathrm{t}
$$

where $\mathrm{k}_{1}\left(\mathrm{~h}^{-1}\right)$ and $\mathrm{k}_{2}\left(\mathrm{~g} \cdot(\mathrm{mg} \cdot \mathrm{h})^{-1}\right)$ are the rate constant of sorption in correctional pseudo-first-order and pseudo-second-order model, respectively; $\mathrm{q}_{\mathrm{e}}\left(\mathrm{mg} \cdot \mathrm{g}^{-1}\right)$ is the amount of SM adsorbed at equilibrium, $\mathrm{q}_{\mathrm{t}}\left(\mathrm{mg} \cdot \mathrm{g}^{-1}\right)$ is the amount of SM adsorbed on the surface of the sorbent at any time.

The linear fitting results are plotted in Figure $5 \mathrm{~b}$ and Figure $5 \mathrm{c}$ with the coefficients of determinations of 0.825 and 0.997 , respectively. The obtained kinetics parameters of the correctional pseudo-first-order kinetics model, and pseudo-second-order kinetics model were $\operatorname{In}\left(\mathrm{q}_{\mathrm{e}}-\mathrm{q}_{\mathrm{t}}\right)=2.93785-0.76482 \mathrm{t} \quad\left(\mathrm{K}_{1}=0.76\right) \quad$ and $\mathrm{t} / \mathrm{q}=0.0014+0.01179 \mathrm{t} \quad\left(\mathrm{K}_{1}=0.09\right), \quad$ respectively. Comparison of the correlation coefficients of the two kinetic models indicates that the pseudo-second-order kinetics model described the data better than the first-order model, which relies on the assumption that adsorption is a chemical reaction, i.e. chemisorption. As reported in the literature [14], quick adsorption of SM on the GO/CNF suggests chemical adsorption. The calculated adsorption capacity $\left(\mathrm{q}_{\mathrm{e}}\right)$ values estimated by the pseudo-second-order model is $85.82 \mathrm{mg} \cdot \mathrm{g}^{-1}$. 

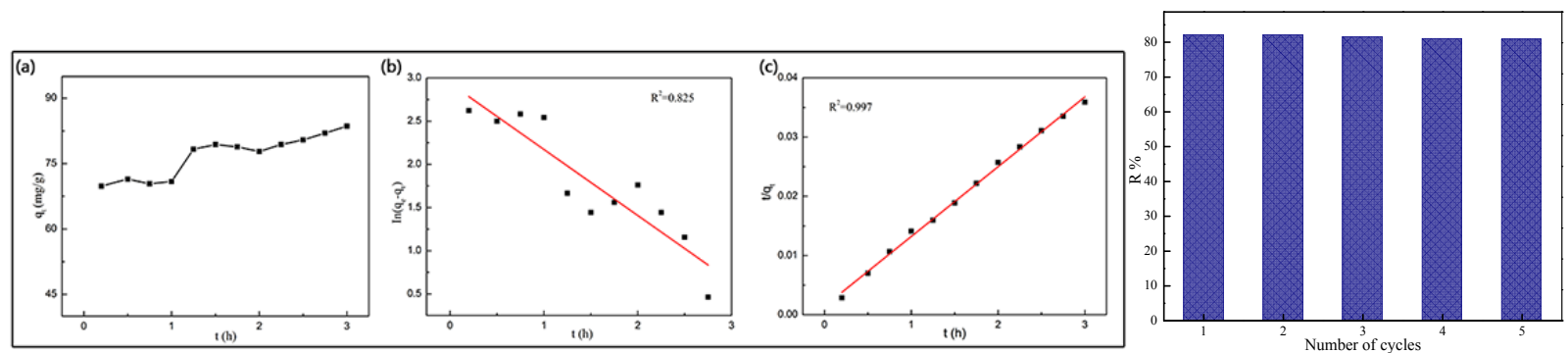

Figure 5 The adsorption kinetics (a) and the pseudo-first-order (b) and pseudo-second-order (c) kinetics models for adsorption of SM on GO/CNF (Left)

Figure 6 Reusability of the GO/CNF for SM removal in five cycles (Right).

Figure 6 shows the regeneration and reusability of the GO/CNF on account of the importance of the recyclability, and economy for adsorbents. The removal percentage (R \%) of SM onto the $\mathrm{GO} / \mathrm{CNF}$ is $81.39 \%$. After five adsorption/ desorption cycles, the $\mathrm{R} \%$ of SM onto the recycled $\mathrm{GO} / \mathrm{CNF}$ aerogel still remains at $77.97 \%$, which only reduces by $3.42 \%$ compared to that of the first cycle. The results indicate that the $\mathrm{GO} / \mathrm{CNF}$ could be still efficiently reused, which illustrates the $\mathrm{GO} / \mathrm{CNF}$ with regeneration performance could be an efficient, economical adsorbent for antibiotics removal.

\section{Conclusions}

In conclusion, a graphene oxide/cellulose nanofibers (GO/CNF) aerogel was prepared by a chemical-ultrasonic process. The GO/CNF has interconnected 3D network structure and the GO/CNF could be still efficiently reused through five regeneration cycles. Consequently, with excellent reusability and outstanding performance in absorption, the $\mathrm{GO} / \mathrm{CNF}$ aerogel could be an efficient, economical and potential adsorbent in antibiotics removal from real wastewater.

\section{Acknowledgements}

Supported by Zhejiang Provincial Natural Science Foundation of China (No. LZ14C160001), Scientific Research Foundation of Zhejiang A\&F University (Grant No. 2014FR077), and Fund for Innovative Research Team of Forestry Engineering Discipline (101-206001000713).

\section{References}

[1] Richardson, S.D. and T.A. Ternes. Analytical chemistry, Water Analy. 2813-2848 (2014), p. 86(6).

[2] Murray, K.E., S.M. Thomas, and A.A. Bodour, Environ. Pollut. 158(12): 3462-3471 (2010).

[3] McKinlay, R., et al. Environ. Pollut. 34(2): 168-183 (2008).

[4] Daughton, C.G. and T.A. Ternes, Environ. Health Per. 107 (Suppl 6): 907 (1999).

[5] Wang, F., et al. Chem. Eng. J. 274: 17-29 (2015).

[6] Zhou, T., et al. Appl.Catal. B- Environ. 136: 294-301 (2013).

[7] Martínez, F., et al. Water Res. 47(15): 5647-5658 (2013).

[8] Ay, F. and F. Kargi. J. Hazard. Mater. 179(1): 622-627 (2010).

[9] Park, S., et al. 2008 ACS Nano. 2(3): 572-578 (2008).

[10]Choi, K.-J., S.-G. Kim, and S.-H. Kim. 2008 J. Hazard. Mater. 151(1): 38-43 (2008).

[11]Chen, J., et al. Carbon. 64: 225-229 (2013).

[12] Olsson, R.T., et al. Nat. Nanotechnol. 5(8): 584-588 (2010).

[13]Fan, Z., et al. Carbon. 48(5): 1686-1689 (2010).

[14]Wang, H., et al. Appl. Surf. Sci. 279: 432-440 (2013). 